\title{
Motion Detection and Face Recognition using Raspberry Pi, as a Part of, the Internet of Things
}

\author{
Zoltán Balogh ${ }^{1}$, Martin Magdin ${ }^{1}$, György Molnár ${ }^{2}$ \\ ${ }^{1}$ Constantine the Philosopher University in Nitra, Faculty of Natural Science, Tr. \\ Andreja Hlinku 1, Nitra, Slovakia, zbalogh@ukf.sk, mmagdin@ukf.sk \\ ${ }^{2}$ Department of Technical Education, Budapest University of Technology and \\ Economics, Budapest, Hungary, molnar.gy@eik.bme.hu
}

\begin{abstract}
Face recognition and motion detection are described in the context of the construction of a system of intelligent solutions, for use in the home, that can be used as a single free standing functional unit or as an element of a bigger system, connected to the Internet of Things. To create a complex system, a micro PC, Raspberry Pi 3, was used together with an application capable of recognizing faces and detecting motion. The outputs are saved into cloud storage, for further processing or archiving. The monitoring system, as designed, can be used autonomously; with the use of a battery and a solar panel, it is possible to place it anywhere. Furthermore, it could be used in various fields, such as health care, for the real-time monitoring of patients, or in the tracking of the spatial activity of people and/or animals. Thus, the system created, may be regarded as a part of the IoT.
\end{abstract}

Keywords: Computer Vision; Raspberry Pi; Face detection; OpenCV; Motion detection

\section{Introduction}

Technological progress has made possible the development of methods of communication between people and objects, facilitating information flow in terms of both speed and security. The Internet of Things (IoT) uses this kind of progress and integration of new elements. As a part of information systems, remote access and various system controls, are enabled.

In accordance with the prevailing philosophy of ever-present communication, including machine to machine communication, the IoT may be defined as a set of technologies designed to allow the connection of heterogeneous objects through various networks and methods of communication. The main objective is to position intelligent devices in different locations to capture, store and manage information, making it is accessible, to anyone anywhere in the world [1]. 
The phrase "Internet of Things" was first used by Kevin Ashton, the founder of the Auto-ID Center in 1999. Although there is no standard definition of "Internet of Things", various technologies (Radio Frequency Identification (RFID), Bar Codes and Global Positioning Systems (GPS)) are used in the implementation of the IoT [2].

The IoT may be regarded as a general term for various technologies, connecting, monitoring and controlling devices such as tags, sensors, actuators, mobile phones, home appliances, vehicles, industrial devices, robots and even medical devices over a data network. There are a number of definitions of and viewpoints regarding the IoT [3] [4].

The concept of the IoT is based on the use of a large number of smart devices, objects and sensors [4] [5]. The sustainable cost and ease of deploying these smart devices is an ever-more critical issue. The lifetime costs of a local IoT installation, "a smart thing", can be divided into three main components: first, the cost of the smart thing itself, hardware and software, and second, the cost of connectivity. Last is the cost of deployment [6].

At first sight, it may seem obvious that the IoT is represented by physical objects. It should, however, be noted that the IoT's value propositions are equally based on the software that runs these. This could present in many forms, for example, embedded, middleware, applications, service composition logic, and management tools [7] [8].

The Internet of Things resulted from new technologies and several complementary technical developments. It provides capabilities that collectively help to bridge the gap between the virtual and physical worlds [1]. These capabilities may include communication and cooperation, addressing capability, identification, perception, information processing, location and user interfaces.

On the basis of the previous claims, the Internet of Things could be considered a simple data network [9]. If this data network is applied to an area, for example, a smart home [10], then it is increasingly being used as a cheap and efficient sensor for simple cameras (IP cameras) [11].

The Internet of Things is the connection of all physical objects based on the Internet infrastructure with the aim of exchanging information; devices and objects are no longer disconnected from the virtual world, but can be remotely controlled and act as points of access to services [1].

Currently, various smart device solutions (gadgets) are being developed. An example might be Intel's new smart bracelet, a system based on the principle of using ultra-sound. The bracelet is designed to detect if the wearer has a fall, and is primarily designed for sick patients with mobility problems). However, one problem with this solution is of its difficulty in detecting that a wearer is paralyzed and needs help (for example, in the case of upper and lower limb paralysis). 
Therefore, at present, a great deal of attention is being paid to solutions that focus on complex motion detection - i.e., to detect limb movement, and also face detection.

According to Mano [12], an increasing number of patients are receiving home treatment. Home treatment has its benefits - especially in the field of psychological support. This is especially true of older people, who often live alone. Older people, however, are do not have the same level of immunity or resistance to various diseases as young people. However, they are not willing to leave the home environment and get to the hospital. Therefore, they are often treated within the framework of home care, but this service often requires 24/7 readiness, in the form of a nurse. This is, however, for most people financially not possible. However, if it is only necessary to keep an eye on the sick person, it is possible to apply elements of the Internet of Things (IoT) with some hope of success. These might be very simple, efficient and inexpensive devices including wireless communication devices and cameras, smartphones or built-in devices e.g. Raspberry Pi. Such technologies are called Health Smart Homes (HSH). HSH is still an area relatively unexplored in the context of the Internet of Things [12].

For all these reasons, we consider the use of Health Smart Homes (HSH) very important [13] [14] [15]. The proposal for the use of HSH results from a combination of the medical environment and the possibilities of implementing information systems in the form of an intelligent home. Such a home is then equipped with a variety of remote sensing devices. The principle of such sensing devices is very simple. The sensing devices send data either at regular intervals and/or in a non-standard (critical) situation. The HSH system is described in detail, for example, in [24] [17], in which the authors propose technological solutions that help caregivers to monitor people in need. The goal is fully fledged medical supervision in the home environment while reducing financial costs [12].

This paper describes the ways of creating a monitoring and detection system with the use of computer vision and Raspberry Pi as a part of the IoT. The IoT may be of service in various fields of everyday life and may be used when detecting motion, face and person recognition. The use of Raspberry Pi and Raspberry Pi with a full HD camera module to create an IoT system is also discussed, with reference to systems that might that be employed in healthcare, security, the education system, industry, etc. The aim of the paper is to create an RPi integrated intelligence with a HD camera that can process and evaluate the scanned image and detect motion. An application has been created that is able to monitor and record the activity of the surroundings with a camera. The monitoring system is capable of recognizing changes in the surroundings. 


\section{Related Work}

The scientific area of computer vision, is currently most popular in the implementation of various applications in daily life. It is already a standard to recognize or localize one or more faces or individual objects.

In the recognition process there are 3 phases: detection, extraction and classification. Detection is used to localize objects in images. Real-time detection of the face with many other objects in the background is not a simple problem. There are situations in which it is not possible to capture the face of an object due to face rotation of more than about $30^{\circ}$, or to a change in the lighting, other factors which might inhibit facial appearance, such as, beards, glasses [18] [19]. The second phase is extraction, and this is determined by certain specifications and requires particular features to detect an object. These features are later used for classification. Classification is the last phase of the recognition process, in which, it is mainly used in the classification of emotional states when the subject of detection is a human face.

In recent years, a lot of literature has appeared dealing with and detailing the various phases of the recognition process [20] [21] [22], or for another view [23].

The research area of computer vision is widely used inHSH. Computer vision in the form of HSH provides various solutions in the form of health and safety services. Recently, computer vision has also become a synonym for marketing and entertainment (Kinnect, though Microsoft has halted its further development) [24] [25].

However, the two fields of face recognition and the IoT are not currently connected. With regard to literature dealing with face detection in IoT, there are very few relevant sources [14] [26] [27]. The problem has a big potential, since the issues of the IoT are currently a 'hot topic' of discussion. Therefore, at least a partial overview of the most recent research works is provided.

According to Kolias et al. [28], for automatic surveillance of a scene in HSH, multiple cameras need to be installed in a given area. However, they must be resistant to current limitations such as noise, lighting conditions, image resolution and computational cost. To overcome such limitations and to increase recognition accuracy, Kolias et al. use a Radio Frequency Identification technology, which is ideal for the unique identification of objects. They examined the feasibility of integrating RFID with hemispheric imaging video cameras. The advantages and limitations of each technology and their integration none the less indicates that their combination could lead to a robust detection of objects and their interactions within an environment. The present work concludes with a presentation of some possible applications of such integration.

Mano et al [12] point to a problem with HSH; according to them, the use of camera and image processing on the IoT is a new research area. The article discusses not only face detection, but also the classification of emotion observable 
on patients' faces. Mano et al. also discuss the existing literature, and show that most of the studies in this area do not put the same effort into monitoring patients. In addition, there are only relatively few studies that take the patient's emotional state into account, and this may well be crucial to their eventual recovery. The result is prototype which runs on multiple computing platforms. Indeed, the present paper was inspired by the ideas formulated by Mano et al.

According Kitajima et al. [11], people today use various smart sensors in the IoT, e.g. wristbands, because these can provide significant amounts of information about health. However, the majority of the information is personal and there are significant concerns that individual privacy could be compromised. If these wristbands are connected to the Internet as an element of the IoT, there is a potential concern about the unintentional leaking of user data.

Most of the studies [14] in the literature focus on the use of body sensors (Galvanic Skin Response (GSR), Electroencephalography (EGG) and other) and the sending of data for future processing using the IoT. The main concern is the loss of data and/or to the data falling into the hands of third parties. A few studies [26] use cameras as smart devices for improving the analysis of the environment (e.g. detecting possible hazardous situations) in HSH. These two different approaches (various sensors implemented in smart wristbands or IP cameras) clearly have advantages and disadvantages and should be used with specific objectives [12].

\section{Materials and Methods}

There is an example of the use of cameras in conjunction with the IoT in the context of the home healthcare presented by Mano in 2016 [12]. A classical wireless architecture for Personal Area Network (WPAN) is used to create a Smart Architecture for In-Home Healthcare (SAHHc) consisting of sensors (IP cameras) and a router with a server (Decision Maker).

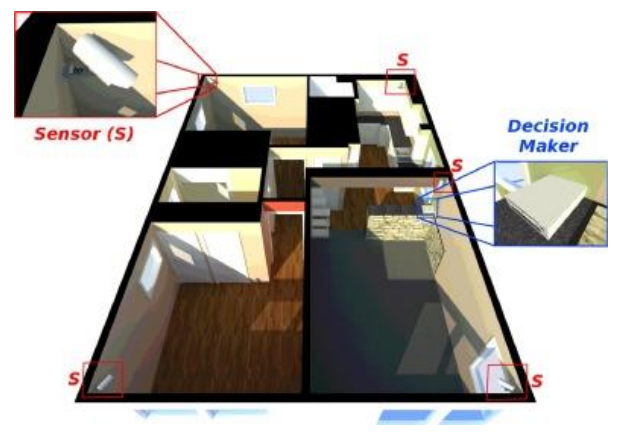

Figure 1

Example of simple SAHHc [19] 
This solution is standard, but from the point of view of security, it carries various risks. Therefore, a classic HD webcam is used, connected it to a Raspberry Pi 3 micro-controller.

The microcontroller Raspberry Pi 3 was the main decision element, the Decision Maker. The communication route is illustrated in the following block scheme:

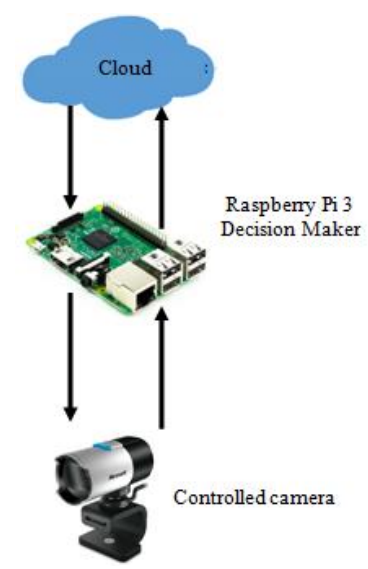

Figure 2

Block scheme of communication

The structure of this system consisted of:

- Simple devices (e.g. in this case HD cameras) that act as sensors and are capable of collecting information about the patient's health and the environment if necessary.

- A decision element, that is a decision maker with more powerful computing resources, in this case, the Raspberry Pi 3.

Sensors can detect the non-standard movement or falls and also capture their faces. When a person enters the room, the sensors detect activity. Depending on how the camera is rotated and the person's face is captured, the person can be identified (conditions depend on the degree of face rotation, light conditions, camera distance, etc.). If the identified person is a patient, the person's activity will start to be monitored. [12].

Face detection, tracking and motion detection is an important and popular research topic in the area of image processing. Computer vision as a scientific and technological study deals with the ability of electronic devices to gather information from a digital image, "to understand a situation" and thus make a decision as to whether to carry out the task or not. This quality is present in technology that is used in all fields of industry and research. Here, the Raspberry Pi 3 micro-controller and the OpenCV pack is described. OpenCV had already been used in previous systems. On the other hand, the hardware design could be 
described together with the software code written in Python, and its main function is person recognition and motion detection. Three programs have been created for facial detection and their functions can be compared on the basis of the algorithms used. The speed and precision of facial and motion recognition can then be compared as well.

\subsection{Raspberry Pi Characteristics}

The Raspberry Pi is a one-chip PC comparable to a (weaker) desktop computer. It contains a port for the screen (HDMI) and via a USB port it is possible to connect it to a keyboard and mouse. Multiple generations of this computer have already been developed, all differing in performance and intended use. The micro CPU is from the ARM family, so it is comparable to a common smartphone. On Raspberry Pi, it is possible to operate various distributions of Linux, Windows 10 or the IoT Core, from Microsoft. Unlike PC Arduino, it is possible to use Raspberry Pi not only for various device control (with GPIO contacts), but also for the relevant application development itself. For facial and motion detection, the newest model of Raspberry Pi 3, model B is used, which was launched in February 2016. It is the first 64-bit Raspberry $\mathrm{Pi}$, and is supplied with in built WiFi and Blue-Tooth (Figure 3).

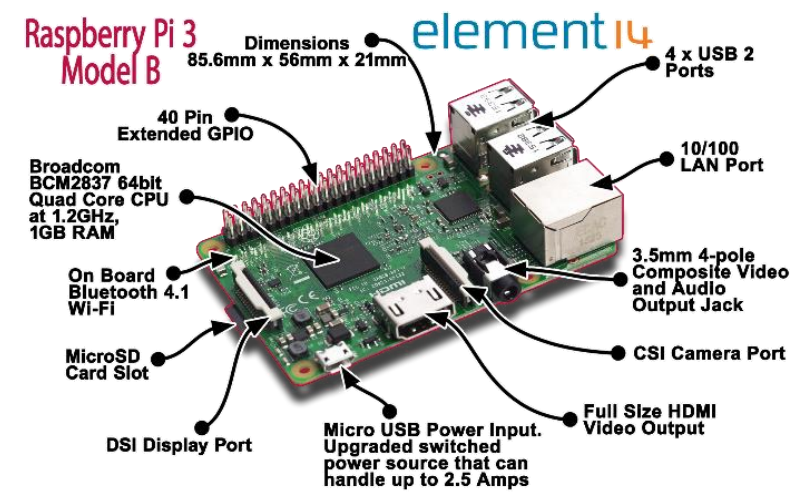

Figure 3

Raspberry Pi 3 Model B

Specifications:

- Quad core 64-bit ARM Cortex A53 with frequency $1.2 \mathrm{GHz}$, approx. $50 \%$ faster than Raspberry Pi 2

- $\quad 802.11 \mathrm{n}$ Wireless LAN

- Bluetooth 4.1 (including Bluetooth Low Energy)

- $\quad 400 \mathrm{MHz}$ Video Core IV multimedia 
- 1 GB LPDDR2-900 SDRAM (900 MHz)

- $100 \mathrm{Mb} / \mathrm{s}$ Ethernet port, 4x USB 2.0, HDMI output

To create an autonomous system, the Raspberry Pi camera module v2 is used. The camera has a high quality 8 megapixel Sony IMX219 image scanner. From the point of view of static images, the camera is capable of making $3280 \times 2464$ image points of static pictures, it also supported 1080p in $30 \mathrm{fps}$, resolution of $720 \mathrm{p}$ in $60 \mathrm{fps}$ and $640 \mathrm{x} 480 \mathrm{p}$ in a 60 or $90 \mathrm{fps}$ video.

Specifications:

- 8 megapixel native resolution

- High quality Sony IMX219 image scanner

- $3280 \times 2464$ image points of static pictures

- Supports 1080p in 30fps, resolution of 720p in $60 \mathrm{fps}$ and $640 x 480 \mathrm{p}$ in a 60 or $90 \mathrm{fps}$ video

- The camera is supported in the newest version of Raspbian

- $\quad 1.4$ um x 1.4 um pixels with technology OmniBSI for high performance (high sensitivity, low crosstalk, low noise)

- Optical size $1 / 4$

- $\quad$ Size: $25 \mathrm{~mm}$ x $23 \mathrm{~mm}$ x $9 \mathrm{~mm}$

- Weight (camera module + connecting cable): $3.4 \mathrm{~g}$ [29]

\subsection{Visual Detection}

Visual detection and facial and object recognition has been one of the biggest challenges in the field of computer vision over the last decade. The potential use of detection systems and object recognition is rather wide, from security systems (identification of authorized users), medical techniques (detection of tumors), industrial applications (visual inspection of products), robotics (navigation of a robot in an inaccessible terrain), to augmented reality, and many others.

Each phase of the recognition process uses various methods and techniques. Detection, extraction and classification have been solved by these methods. Over time, not only new methods have been developed but also algorithms have developed from the original methods, until currently, there are over 200. According to this approach and the its various uses, detection methods can be divided into four categories [30]:

- Knowledge-based methods

- Feature invariant approaches 
- $\quad$ Template matching

- Appearance-based methods

The division of the methods is not uniform, because the methods for all three phases of the recognition process could be used. From the point of view of speed and simplicity of use, an OpenCV library that includes a powerful Viola-Jones detector based on Haar cascades might be suggested (for more information see [31]).

\subsection{OpenCV}

Open CV (Open-source Computer Vision, opencv.org) might be called the Swiss army knife of computer vision. It has a range of modules by which it is possible to solve a number of problems in computer vision. The most useful part of OpenCV may well be its architecture and memory management. It provides a framework within which it is possible to work with images and videos in any number of ways. The algorithms to recognize faces are accessible in OpenCV library and are the following:

- FaceRecognizer.Eigenfaces: Eigenfaces, also described as PCA, first used by Turk and Pentland in 1991

- FaceRecognizer.Fisherfaces: Fisherfaces, also described as LDA, invented by Belhumeur, Hespanha and Kriegman in 1997 [32]

- FaceRecognizer.LBPH: Local Binary Pattern Histograms, invented by Ahonen, Hadid and Pietikäinen in 2004 [33]

The choice of Fisherfaces has been made because it was based on the LDA algorithm. Rashmi [34] claims that when comparing different algorithms, a 95.3\% success was achieved with LDA, while the time needed for detection was compared to other algorithms. The method used in Fisherfaces is taught from a class transformation matrix. Unlike the Eigenfaces method, it does not record the intensity of lighting. The discriminatory analysis finds the facial traits needed for person comparison. It is necessary to mention that the Fisherfaces' performance is influenced to a great extent by input data. In practical terms, if in a specific case Fisherfaces is taught using a well-lighted image, then in experiments with bad lighting, there will be a higher number of incorrect results. this is logical, as the method does not have a chance to capture the lighting on the images. The Fisherfaces algorithm is described below.

$\mathrm{X}$ is a random vector from the c class:

$$
\begin{aligned}
& X=\left\{X_{1}, X_{2}, . ., X_{c}\right\} \\
& X_{i}=\left\{X_{1}, X_{2}, . ., X_{c}\right\}
\end{aligned}
$$


The scattering of the matrices SB and SW are calculated as:

$$
\begin{aligned}
& S_{B}=\sum_{i=1}^{c} N_{i}\left(\mu_{i}-\mu\right)\left(\mu_{i}-\mu\right)^{T} \\
& S_{W}=\sum_{i=1}^{c} \sum x_{j} \in x_{i}\left(x_{j}-\mu_{i}\right)\left(x_{j}-\mu_{i}\right)^{T}
\end{aligned}
$$

Where $\mu$ is the total mean:

$$
\mu=\frac{1}{N} \sum_{i=1}^{N} X_{i}
$$

And $\mu \mathrm{i}$ is the mean of class $\mathrm{i} \epsilon\{1, \ldots, \mathrm{c}\}$ :

$$
\mu_{i}=\frac{1}{\left|X_{i}\right|} \sum X_{j} \in X_{i}^{X_{j}}
$$

Fisher's standard algorithm searches for the projection of $\mathrm{W}$ that maximizes the criterion of divisibility of the given class [35] [32]:

$$
W=W_{f l d}^{T} W_{p c a}^{T}
$$

These kinds of solutions have been used in various industrial applications, in educational projects, and more often in intelligent households. Facial recognition and motion detection have been used to construct a system of intelligent solutions in households that can be used as a separate functional unit or as an element of a bigger system connected to a technology of the Internet of Things.

\section{An experiment and a discussion of Face Recognition and Motion Detection}

The OpenCV library was used for face recognition and motion detection. It is a multi-platform library of programming functions related to computer vision. First, a proper choice of algorithm for face recognition must be determined. OpenCV contains two popular methods for facial recognition, the Haar Cascade and Local Binary Patterns (LBP). The Haar Cascade method is based on the principle of machine learning. The cascade function is trained using multiple images and is then used to detect objects in other images. The LBP Method (local binary pattern) is used to describe the characteristics of images with various attributes that characterize the image. When creating an attribute, it goes through each pixel of the image and using assessing features arrives at a value. In this case, the LBP 
method was because its functions were simpler and faster than the Haar Cascade method.

Both algorithms use XML files to record the properties of the object which needs to be detected. The OpenCV library already contains some XML files for face recognition and body detection. Using this, a particular LBP or Haar Cascade XML file is created via training. It is possible to train the classifier to detect any object that may be required. Three programs working in different ways were created to detect faces. This means that multiple methods were tried out in order to compare the programs with respect to speed and accuracy.

\subsection{Face Recognition}

\section{Face_recognition.py}

The first program was slow (5 - 6 FPS), but worked very reliably. It uses a single core processor for processing images taken by the Raspberry Pi camera module. The algorithm was programmed using the OpenCV library, and employed a cascading file "lbpcascades_frontalface.xml" to recognize the faces in the picture (Figure 4).

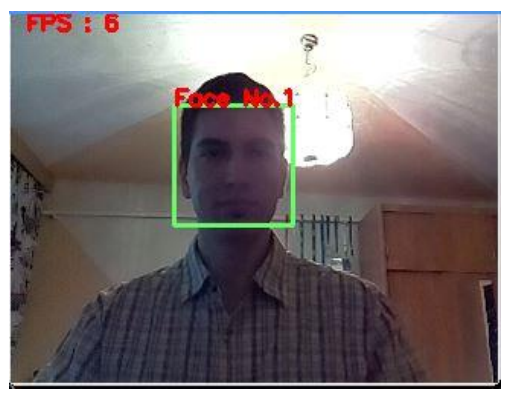

Figure 4

Face detection by "lbpcascades_frontalface.xml"

As the program runs, when it recognizes a face, this is indicated by a green square appearing around the face. Given that only one core was used, FPS was quite slow, showing only 5-6 frames per second and using about 53 percent of system resources.

\section{Object_recognition.py}

The second program could recognize more faces and other objects, such as mobile phones, at the same time. The algorithm worked in the same way as the previous face_recognition.py program but another cascade file cascade.xml was used here for the detection of mobile phones. It should be emphasized that the XML file used to detect phones was generated by Radames Ajna and Thiago Hersan. The output of the program is shown in the figure below (Figure 5). 


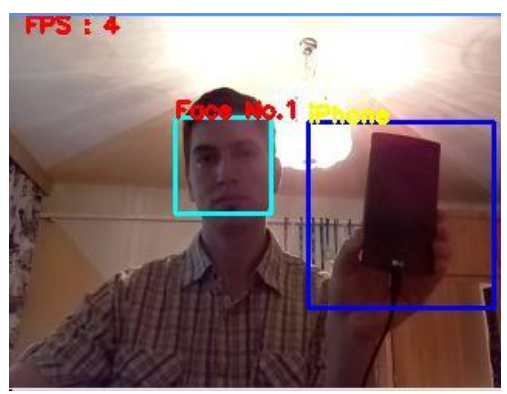

Figure 5

Detection of mobile phones

In this program, two different cascading files are used for identification: cascade.xml for phone detection and lbpcascades_frontalface.xml for face detection. This means that the search time is doubled and the FPS rate drops to 3 4 images per second, while the program uses about 61 percent of system resources.

\section{Multi_core_face_recognition.py}

The previous two programs were slowed down, achieving only four to six FPS and therefore are not the best solution to the problem. In this case, more cores could have been used, seeing as the Raspberry Pi 3 contains four cores. In cases where it is necessary for the program to work faster, the algorithms for facial recognition need to be made to operate in parallel. A library for Python can be imported so that multiple processes can operate at once, as library features could be used to support parallel processing (multiprocessing). This program loads four images at once (one for each core) to be processed using a face recognition algorithm and it finally evaluates the results. Using the parallel processing, the program reached rates of up to 15 FPS, about three times faster than in the two previous programs, and used about 91 percent of system resources. The output of the program is shown in the figure below (Figure 6).

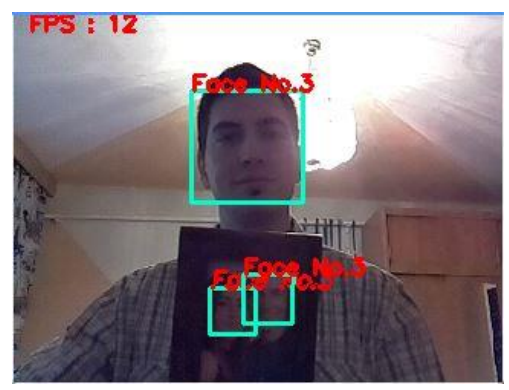

Figure 6

Face detection using four cores of Raspberry Pi 3 
This program is similar to the first one (face_recognition.py), with the exception that in this case all four cores are used and thus the program works faster and more smoothly.

Table 1

The use of system resources

\begin{tabular}{cccc}
\hline Program name & $\begin{array}{c}\text { The } \\
\text { number } \\
\text { of used } \\
\text { cores }\end{array}$ & FPS & $\begin{array}{c}\text { System } \\
\text { resources }\end{array}$ \\
\hline face_recognition.py & 1 & 6 & $53.00 \%$ \\
object_recognition.py & 1 & 4 & $45.00 \%$ \\
multi_core_face_recognition.py & 4 & 15 & $91.00 \%$ \\
\hline
\end{tabular}

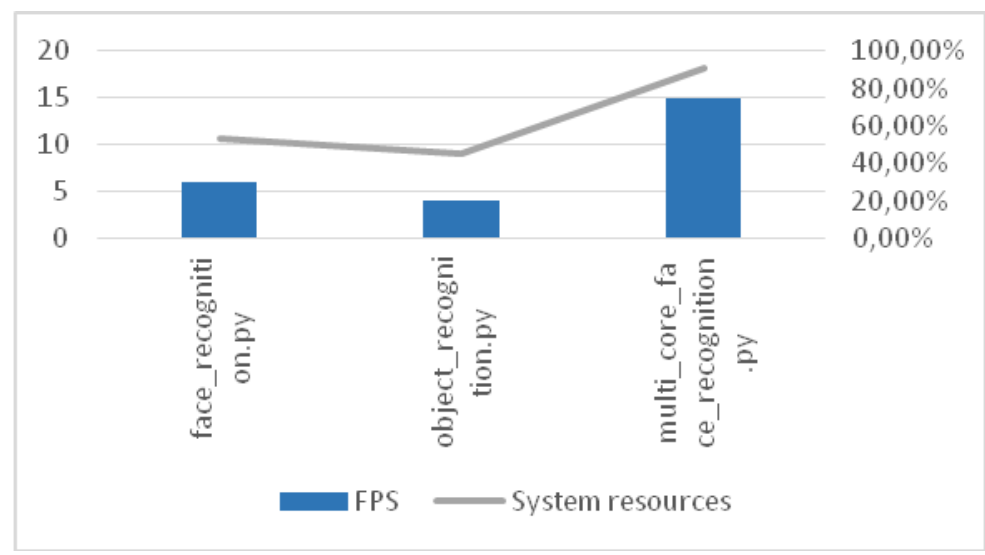

Figure 7

The use of system resources

In Table 1 and Figure 7, the FPS value and use of system resources were directly proportional, which means that if more FPS were needed, then Raspberry had to use more system resources (cores).

\subsection{Motion Detection}

For detecting motion, two programs were created. The first program (detektor_pohybu.py) works without using OpenCV library and when it perceives a movement it creates an image and stores it in a folder. The second program (monitorovaci_system.py) uses an OpenCV library and after detecting motion it draws a green box around the detected area and records the file into Dropbox. 
The programs were tested in internal and external environments for 5 hours in each case. The motion detector saved 72 images outside and 56 inside, 128 altogether. There were 109 good images of all the results on which real movement could be detected. The other 19 pictures showed distorted results, for example due to changing light conditions. In the course of the testing of the monitoring system, it saved 64 images outside and 48 inside, 112 altogether. There were 107 good images of all the results and 5 distorted ones. The results may be seen in the table and graph below.

Table 2

Testing of motion detection and monitoring system

\begin{tabular}{ccccccc}
\hline & Time & Inside & Outside & Together & accurate & inaccurate \\
\hline motion detector & 5 & 56 & 72 & 128 & 109 & 19 \\
$\begin{array}{c}\text { hour } \\
\text { system }\end{array}$ & $\begin{array}{c}5 \\
\text { hour }\end{array}$ & 48 & 64 & 112 & 107 & 5 \\
\hline
\end{tabular}

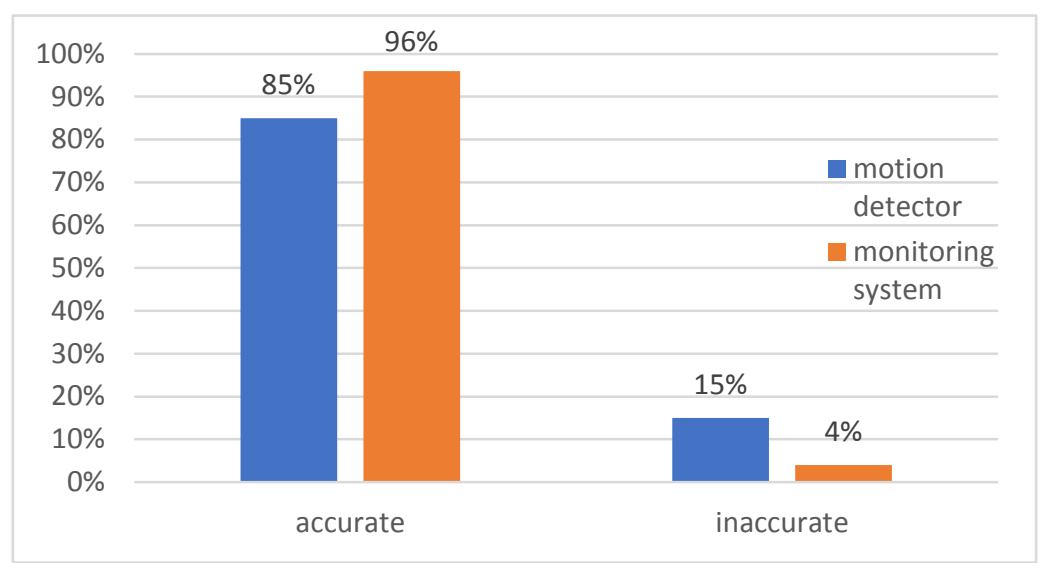

Figure 8

Percentage of accurate and inaccurate image while recording

As may be seen in Figure 8, the monitoring system achieved better results because it took the weighted mean of images for comparison, instead of the previous images. Thus, if the lighting conditions change, the algorithm still works precisely.

A functioning system for monitoring internal and external environments was designed, and was capable of being implemented in practice as an intelligent security system or to monitor different rooms, objects, people or animals in nature. The camera recorded only when motion was perceived and only then stored or sent images to cloud storage. In this way, space on the hard drive could be saved, 
since instead of watching long videos, pictures were taken, and if an important image (for example, showing a burglary) needed to be found, it would be enough to search by date and see what happened in the monitored area.

\section{Conclusions}

The Internet of Things is a new trend in informatics that connects different devices to the Internet, usually wirelessly, using Wi-Fi or Bluetooth. The monitoring system designed here, could also be used autonomously, meaning that with the help of a battery or a solar panel, it could be placed anywhere and would operate without any outside control. It could be used in various fields, such as healthcare, monitoring patients in hospitals and checking their status in real time. Motion activity could be monitored, for example, in parking lots, allowing access to the lot only if there are vacancies. Then the activity and behavior of animals can be observed and described, using the obtained data as detailed in previous publications of the author of the present and other related paper [36] [37] [38] or as an intelligent safety and mobile system, whether to monitor different rooms or animals in the wild.

However, even though cameras can provide significant amounts of information, the vast majority is personal data, and there are significant concerns that individual privacy could be compromised. Furthermore, since home appliances are increasingly being connected to the Internet via the IoT, it has become possible for user images to leak out unintentionally. With these concerns in mind, there is a need to propose a human detection method that protects user privacy by using intentionally blurred images. In this method, the presence of a human being is determined by dividing an image into several regions and then calculating the heart rate detected in each region. This proposal was realized first by Kitajima et al. in 2017. In overall performance evaluation, the proposed method showed favorable performance results when compared with an OpenCV based face detection method, and was confirmed to be an effective method for detecting human beings in both normal and blurred images [11]. Therefore, in future research, the focus will be on the application of the method, in practice.

\section{References}

[1] J. I. R. Molano, D. Betancourt, G. Gómez, "Internet of things: A prototype architecture using a raspberry $\mathrm{Pi}$," Lecture Notes in Business Information Processing, Vol. 224, 2015, pp. 618-631

[2] L. G. Guo, Y. R. Huang, J. Cai, L. G. Qu, "Investigation of architecture, key technology and application strategy for the internet of things," Proceedings of 2011 Cross Strait Quad-Regional Radio Science and Wireless Technology Conference, CSQRWC 2011, 2011, pp. 1196-1199

[3] L., Atzori, A. Iera, G. Morabito, "The Internet of Things: A survey. Computer Networks 54(15), 2010, pp. 2787-2805, doi:10.1016/j.comnet.2010.05.010 
[4] J. Katona, T. Ujbanyi, G. Sziladi, A. Kovari: Electroencephalogram-Based Brain-Computer Interface for Internet of Robotic Things, Cognitive Infocommunications, Theory and Applications, Springer 2018, pp. 253-275

[5] J. Katona, P. Dukan, T. Ujbanyi, A. Kovari: Control of incoming calls by a windows phone based brain computer interface, Proceedings of the 15th IEEE International Symposium on Computational Intelligence and Informatics, Budapest, Hungary, 2014, pp. 121-125

[6] A. Iivari, J. Koivusaari, H. Ailisto,“A rapid deployment solution prototype for IoT devices," Lecture Notes in Computer Science (including subseries Lecture Notes in Artificial Intelligence and Lecture Notes in Bioinformatics), Vol. 10070 LNCS, 2016, pp. 275-283

[7] C. Ebert, C. Jones, "Embedded software: Facts, figures, and future," Computer, 42(4), 2009, pp. 42-52, doi:10.1109/MC.2009.118

[8] S. Stastny, B.A Farshchian, T. Vilarinho, "Designing an application store for the internet of things: Requirements and challenges," Lecture Notes in Computer Science (including subseries Lecture Notes in Artificial Intelligence and Lecture Notes in Bioinformatics), Vol. 9425, 2015, pp. 313-327

[9] I. Farkas, P. Dukan, J. Katona, A. Kovari: Wireless sensor network protocol developed for microcontroller based Wireless Sensor units, and data processing with visualization by LabVIEW, Proceedings of the IEEE $12^{\text {th }}$ International Symposium on Applied Machine Intelligence and Informatics, Herlany, Slovakia, 2014, pp. 95-98

[10] P. Dukan, A. Kovari: Cloud-based smart metering system, Proceeding of the $14^{\text {th }}$ IEEE International Symposium on Computational Intelligence and Informatics, Budapest, Hungary, 2013, pp. 499-502

[11] T. Kitajima, E. A. Y. Murakami, S. Yoshimoto, Y. Kuroda, \& O. Oshiro, "Human detection using biological signals in camera images with privacy aware," Proceedings of the 16th International Conference on Intelligent Systems Design and Applications, Volume 557, 2017, pp. 175-186, doi:10.1007/978-3-319-53480-0_18

[12] L. Y. Mano, B. S. Faiçal, L. H. V. Nakamura, P. H. Gomes, G. L. Libralon, R. I. Meneguete, J. Ueyama, "Exploiting IoT technologies for enhancing health smart homes through patient identification and emotion recognition," Computer Communications, 89-90, 2016, pp. 178-190, doi:10.1016/j.comcom.2016.03.010

[13] G. Riva, "Ambient intelligence in health care," Cyberpsychology and Behavior, 6(3), 2003, pp. 295-300, doi:10.1089/109493103322011597

[14] E. Romero, A,. Araujo, J. M. Moya, J.-M. de Goyeneche, J. C. Vallejo, P. Malagon, D. Villanueva, D. Faga, "Image processing based services for ambient assistant scenarios,"Distributed Computing, Artificial Intelligence, 
Bioinformatics, Soft Computing, and Ambient Assisted Living. Lecture Notes in Computer Science, 5518, Springer, Berlin, Heidelberg, 2009, pp. 800-807

[15] A. N. Siriwardena, "Current state and future possibilities for ambient intelligence to support improvements in the quality of health and social care," Quality in Primary Care, 17(6), 2009, pp. 373-375

[16] V. Rialle, F. Duchene, N. Noury, L. Bajolle, J. Demongeot. , "Health Smart home: information technology for patients at home,"Telemed. J. e-Health, 8 (4), 2002, pp. 395-409

[17] J. A. Stankovic, Q. Cao, T. Doan, L. Fang, Z. He, R. Kiran, S. Lin, S. Son, R. Stoleru, A. Wood, "Wireless sensor networks for in-home healthcare: potential and challenges,"High Confidence Medical Device Software and Systems Workshop (HCMDSS), 2005

[18] Y. L. Tian, T. Kanade, C. J. F. Handbook of Face Recognition. Springer, 2005

[19] M. Magdin, M. Turcani, L. Hudec, "Evaluating the Emotional State of a User Using a Webcam," International Journal of Interactive Multimedia and Artificial Intelligence. 4(1), Special Issue: SI, 2016, pp. 61-68

[20] K. Bahreini, R. Nadolski, \& W. Westera, "Towards multimodal emotion recognition in e-learning environments," Interactive Learning Environments, no. Ahead-of-print, 2014, pp. 1-16

[21] U. Bakshi, \& R. Singhal, "A Survey of face detection methods and feature extraction techniques of face recognition,"International Journal of Emerging Trends \& Technology in Computer Science (IJETTCS), 3(3), 2014, pp. 233-237

[22] V. Bettadapura, "Face Expression Recognition and Analysis: The State of the Art,"arXiv: Tech Report, (4), 2012, pp. 1-27

[23] A. Ollo-López, \& M. E. Aramendía-Muneta, "ICT impact on competitiveness, innovation and environment,"Telematics and Informatics, 29 (2), 2012, pp. 204-210

[24] S. Marcutti, G. V. Vercelli, "Enabling touchless interfaces for mobile platform: State of the art and future trends," Lecture Notes in Computer Science (including subseries Lecture Notes in Artificial Intelligence and Lecture Notes in Bioinformatics), Vol. 9769, 2016, pp. 251-260

[25] A. Chaudhary, R. Klette, J. L. Raheja, X. Jin, "Introduction to the special issue on computer vision in road safety and intelligent traffic," Eurasip Journal on Image and Video Processing, 2017(1), 2017, doi:10.1186/s13640-017-0166-5

[26] J. Augusto, P. McCullagh, V. McClelland, J. Walkden, "Enhanced healthcare provision through assisted decision-making in a smart home 
environment,"IEEE Second Workshop on Artificial Inteligence Techniques for Ambient Inteligence, IEEE Computer Society, 2007

[27] S. Helal, W. Mann, J. King, Y. Kaddoura, E. Jansen.., "The gator tech smart house: a programmable pervasive space,"Computer, 38 (3), 2005, pp. $50-60$

[28] V. Kolias, I. Giannoukos, C. Anagnostopoulos, I. Anagnostopoulos, V. Loumos, \& E. Kayafas, "Integrating RFID on event-based hemispheric imaging for internet of things assistive applications," Paper presented at the ACM International Conference Proceeding Series, 2010, doi:10.1145/1839294.1839367

[29] H. Tubman, Raspberry Pi Camera Board. https://www.adafruit.com/products/1367l, 2016

[30] M. Yang, D. J. Kriegman \& N. Ahuja, "Detecting faces in images: A survey," IEEE Transactions on Pattern Analysis and Machine Intelligence, 24(1), 2002, pp. 34-58. doi:10.1109/34.982883

[31] P. Viola \& M. J. Jones, "Robust real-time face detection," International Journal of Computer Vision, 57(2), 2004, pp. 137-154, doi:10.1023/B:VISI.0000013087.49260.fb

[32] P. N. Belhumeur, J. P. Hespanha, D. J. Kriegman, "Eigenfaces vs. fisherfaces: Recognition using class specific linear projection," IEEE Trans Pattern Anal Mach Intell, 19(7), 1997, pp. 711-720, doi:10.1109/34.598228

[33] D. Baggio, S. Emami, D. Escrivá, K. Ievgen, N. Mahmood, J. Saragih \& R. Shilkrot,"Mastering OpenCV with practical computer vision projects," Packt Publishing Ltd, 2012

[34] R. Rashmi, D. Namrata, "A Review on Comparison of Face Recognition Algorithm Based on Their Accuracy Rate," International Journal of Computer Sciences and Engineering, 3(2), 2015, pp. 40-44

[35] P. N. Belhumeur, J. P. Hespanha, D. J. Kriegman, "Eigenfaces vs. Fisherfaces: Recognition using class specific linear projection," In: Buxton, B., Cipolla, R. (eds.) $4^{\text {th }}$ European Conference on Computer Vision, ECCV 1996, Vol. 1064, 1996, pp. 45-58

[36] Z. Balogh, R. Bízik, M. Turčáni, Š. Koprda, "Proposal for Spatial Monitoring Activities Using the Raspberry Pi and LF RFID Technology," In: Zeng, Q.-A. (ed.) Wireless Communications, Networking and Applications, Vol. 348, Lecture Notes in Electrical Engineering, 2016, pp. 641-651

[37] Z. Balogh, M. Turčáni, "Complex design of monitoring system for small animals by the use of micro PC and RFID technology," In: Oualkadi, A. E., Moussati, A. E., Choubani, F. (eds.) Mediterranean Conference on 
Information and Communication Technologies, MedCT 2015, Vol. 380, 2016, pp. 55-63

[38] R. Pinter, S. Maravić Čisar, "Measuring Team Member Performance in Project Based Learning" Journal of Applied Technical and Educational Sciences, ST Press, 2018, Volume 8, Issue 4, pp. 22-34 